International Journal of Computer Networks \& Communications (IJCNC) Vol.4, No.3, May 2012

\title{
MUlTILAYERED BOW-TIE ANTENNAS DESIGN FOR RFID AND RADAR APPLICATIONS USING A SIMPLE EQUiVALENT TRANSMISSION LINE MODEL
}

\author{
S. Didouh, M. Abri and F. T. Bendimerad \\ Telecommunications Laboratory, Faculty of Technology \\ Abou-Bekr Belkaïd University, Tlemcen, 13000, Algeria \\ Email: dsomaia_telecomehotmail.fr
}

\begin{abstract}
In this paper we propose the design of bow-tie antenna based on a simple modified transmission line model. The developed model is simple, accurate and takes into account all the antenna characteristics. The simulated antennas are designed to resonate at the frequencies $2.4 \mathrm{GHz}$ and $8 \mathrm{GHz}$ corresponding to RFID and Radars applications. To validate this last the obtained simulation results are compared to those obtained by the moment's method (Agilent Momentum software). Using this transmission line approach the resonant frequency, input impedance, return loss can be determined simultaneously. The paper reports several simulation results that confirm the validity of the developed model. The obtained results are then presented and discussed.
\end{abstract}

\section{KEY WORDS}

Bow-tie antenna, transmission line model, moment's method (Momentum), aperture coupled feed.

\section{INTRODUCTION}

In recent years, microstrip antennas are popular and getting more and more attention due to their excellent advantages such as light weight, thin profile configurations, low cost of fabrication, reliability, conformal structure and ease of fabrication [1-2]. Microstrip antennas have different geometrical shapes which are used depending upon the applications. Actually, researchers are interested in the design and development of compact microstip radiating elements such as Bowtie microstip antenna [3].

In 1996, the bow-tie structure was first proposed by George and al [4]. A bow-tie patch is the combination of imaginary image of two triangular patches which are fabricated in a single substrate. This type of antenna is used extensively in many applications such as ground penetrating radar [5-6], wireless communications and mobile station [7-9].

The printed antennas characterization requires the use of software using rigorous numerical methods such as integral equations solved by the moment's method. These we offer precision course, but they require tedious calculations. Using the transmission line model provides the best solution. In the present paper, an attempt was made to design a bow-tie antenna by the equivalent model based on the transmission line model which makes it possible to take in account the whole antennas geometrical, electrical characteristics.

The paper reports several simulation results that confirm the validity of the developed model. The obtained results are then presented and discussed. 
International Journal of Computer Networks \& Communications (IJCNC) Vol.4, No.3, May 2012

\section{TRANSMISSION LINE MODEL}

This model considers the patch as a transmission line of width $\mathrm{W}$ with two radiating slots on each end. For a desired frequency $f_{0}$, the width $\mathrm{W}$ can be estimated using [10]:

$$
\mathrm{W}=\frac{\lambda_{0}}{2} \sqrt{\frac{2}{\varepsilon_{\mathrm{r}}+1}}
$$

Where:

$\lambda_{0}$ is free space wavelength.

The microstrip patch has an inhomogeneous configuration (air above and dielectric below), which can be replaced by a homogeneous configuration. This is done by introducing a new medium with an effective permittivity $\left(1<\varepsilon_{\text {reff }}<\varepsilon_{\mathrm{r}}\right)$, which has the same electrical characteristics (impedance and phase velocity) as the original medium.

For $\frac{\mathrm{W}}{\mathrm{h}}>1$, the effective dielectric constant is [11]:

$\varepsilon_{\text {reff }}=\frac{\varepsilon_{\mathrm{r}}+1}{2}+\frac{\varepsilon_{\mathrm{r}}-1}{2}\left[1+12 \frac{\mathrm{h}}{\mathrm{W}}\right]^{\frac{-1}{2}}$

Where $\mathrm{W}$ is the patch width, $h$ is the height of substrate and $\varepsilon_{\mathrm{r}}$ is the dielectric constant of the substrate.

The fringing fields around the edges of the patch as shown in figure 1 (a) make it appear electrically larger than its physical length by $2 \Delta \mathrm{L}$. This size increase can be seen in figure 1 (b).

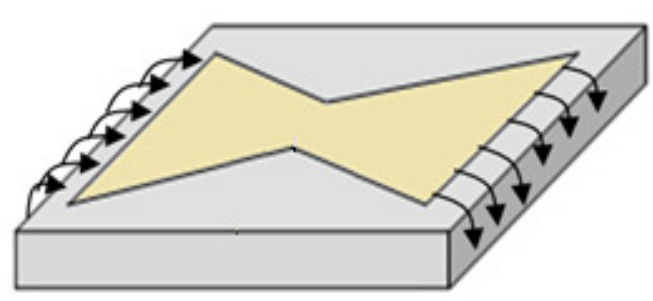

(a)

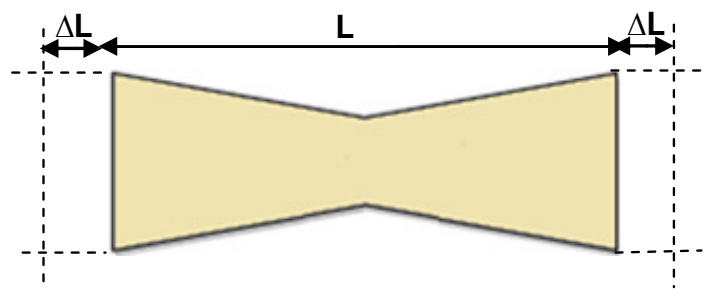

(b)

Figure 1. Bow-tie antenna and its effective length. 
International Journal of Computer Networks \& Communications (IJCNC) Vol.4, No.3, May 2012

The effective patch length can be written in terms of effective dielectric constant as:

$$
\begin{aligned}
& \frac{\Delta \mathrm{L}}{\mathrm{h}}=0.412 \frac{\left(\varepsilon_{\text {reff }}+0.3\right)\left(\frac{\mathrm{W}}{\mathrm{h}}+0.264\right)}{\left(\varepsilon_{\text {reff }}-0.258\right)\left(\frac{\mathrm{W}}{\mathrm{h}}+0.8\right)} \\
& \mathrm{L}_{\text {eff }}=\mathrm{L}+2 \Delta \mathrm{L}
\end{aligned}
$$

The resonant frequency $\mathrm{f}_{0}$ (for dominant mode $\mathrm{TM}_{10}$ ) is:

$$
\mathrm{f}_{0}=\frac{\mathrm{c}}{2 \mathrm{~L}_{\text {eff }} \sqrt{\varepsilon_{\text {eff }}}}
$$

Where $c$ is the speed of light in free space.

\section{APERTURE COUPLED-FED MICROSTRIP BOW-TIE ANTENNA}

The antenna element is formed by two dielectric substrate layers separated in the middle by a ground plane. The radiating patch element is photo-etched on the upper substrate and this is known as the antenna substrate. The aperture or slot is placed on the ground plane [11]. In this work, our choice was made primarily on rectangular slot.

The geometry of a basic aperture-coupled microstrip bow-tie antenna is shown in figure 2 .

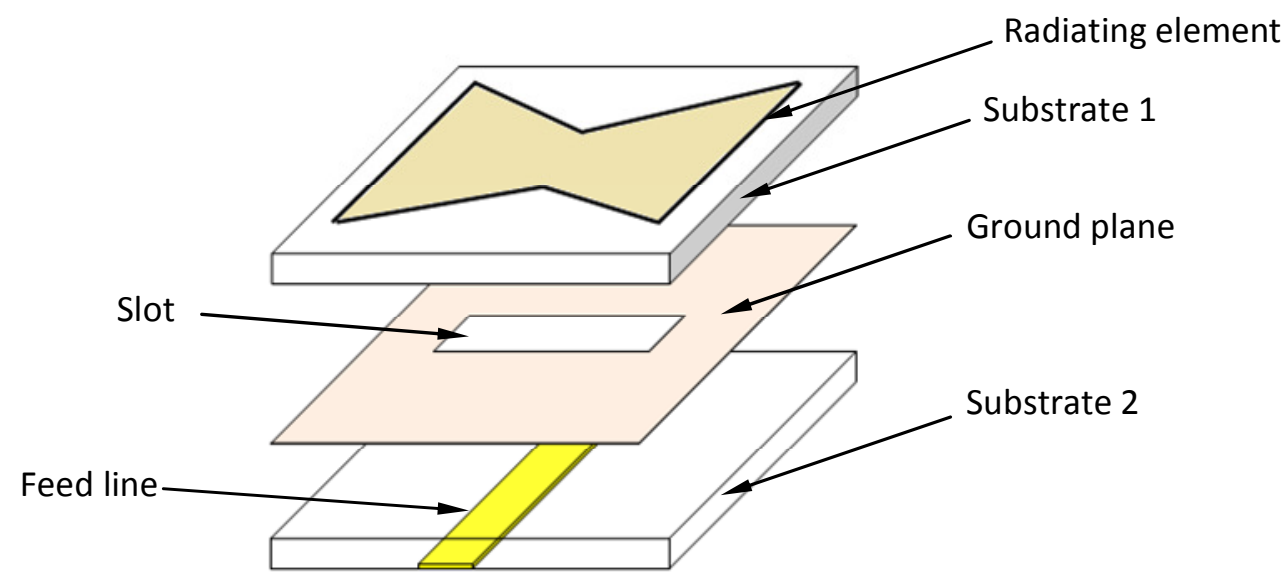

Figure 2. Configuration of bow-tie antenna fed by Aperture-coupled.

The proposed transmission line equivalent circuit for an aperture coupled bow-tie antenna fed via microstrip line is shown in figure 3 . 
International Journal of Computer Networks \& Communications (IJCNC) Vol.4, No.3, May 2012

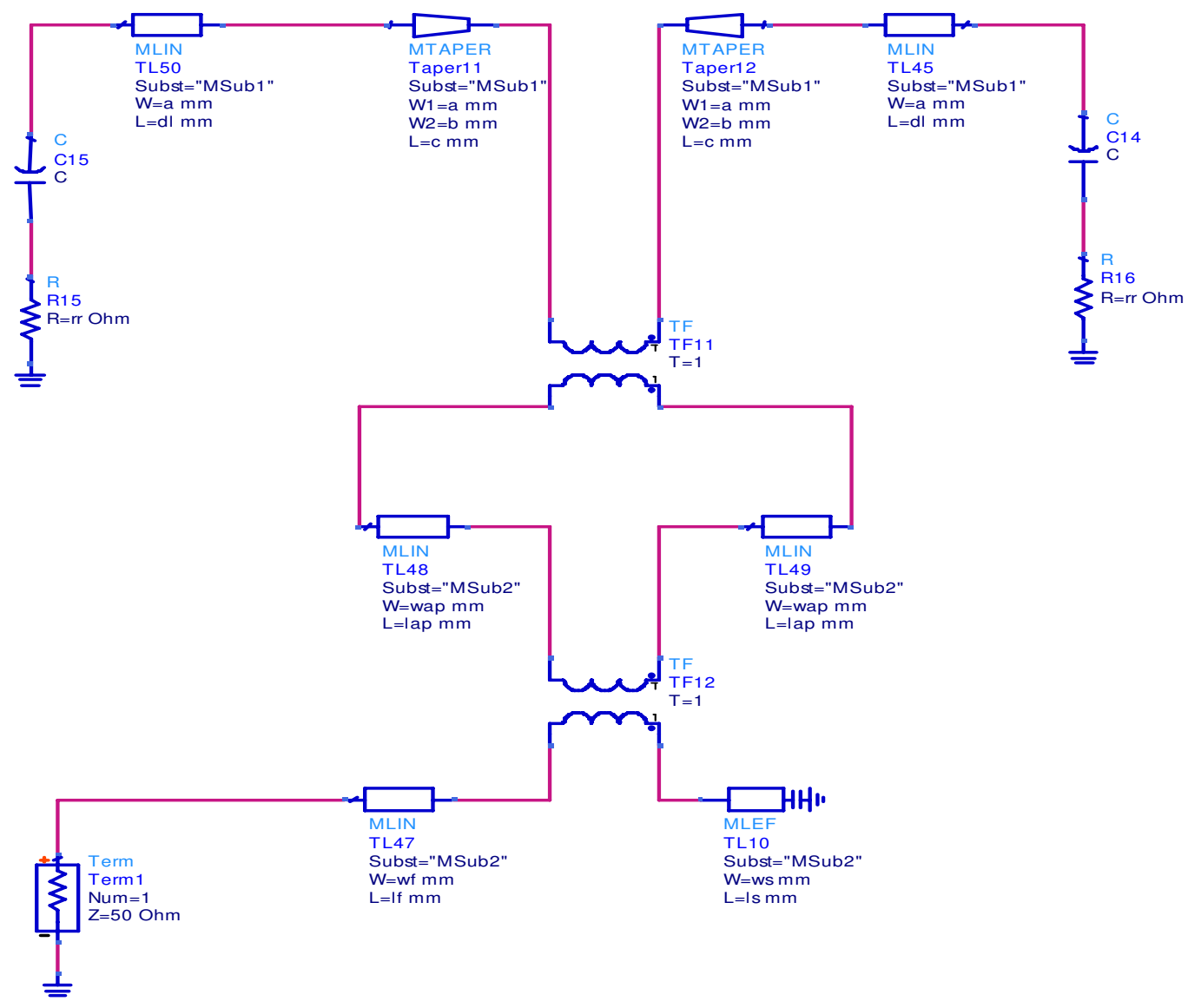

Figure 3. Equivalent circuit of the proposed antenna.

In this equivalent circuit, two ideal transformers are assumed between the slot ground plane and both sides of the line. The energy is transferred and store in these two transformers in term of load susceptance. In fact, all the energy is passed the slot aperture, and deliver to the patch for radiating. The ratios of these two transformers can be determined using [11] :

$\mathrm{n}_{1}=\frac{\mathrm{L}_{\mathrm{a}}}{\mathrm{L}}$

Where $L_{a}$ is the length of the slot.

While the second transformation ratio $\mathrm{n}_{2}$ can be approximated by the expression :

$\mathrm{n}_{2}=\frac{\mathrm{L}_{\mathrm{a}}}{\sqrt{\mathrm{W} \cdot \mathrm{h}}}$

To determine the capacitance $\mathrm{C}$ we use the formula of $\mathrm{C}_{\mathrm{dyn}}[10]$. 


$$
\begin{aligned}
& C(\varepsilon)=\frac{\varepsilon_{0} \varepsilon_{\mathrm{r}} \mathrm{A}}{\mathrm{h} \gamma_{\mathrm{n}} \gamma_{\mathrm{m}}}+\frac{1}{2 \gamma_{\mathrm{n}}}\left(\frac{\varepsilon_{\text {reff }}\left(\varepsilon_{\mathrm{r}}, \mathrm{h}, \mathrm{W}\right)}{\mathrm{c}_{0} \mathrm{Z}\left(\varepsilon_{\mathrm{r}}=1, \mathrm{~h}, \mathrm{~W}\right)}\right)-\frac{\varepsilon_{0} \varepsilon_{\mathrm{r}} \mathrm{A}}{\mathrm{h}} \\
& \gamma_{\mathrm{j}}=\left\{\begin{array}{l}
1, \mathrm{j}=0 \\
2, \mathrm{j} \neq 0
\end{array}\right.
\end{aligned}
$$

\section{RESULTS AND DISCUSSIONS}

The validity of the suggested model is highlighted by comparing the results of the return loss, the input phase, input antenna VSWR and input impedance locus to those obtained by the moment's method of the Momentum Software. The simulated antennas are designed to resonate respectively at the frequencies $2.4 \mathrm{GHz}$ and $8 \mathrm{GHz}$ corresponding to RFID and Radar applications.

\section{1. Multi-layered bow-tie antenna operating at the resonant frequency $2.4 \mathrm{GHz}$}

Using the transmission line model, the antenna was optimized to operate et the resonant frequency of $2.4 \mathrm{GHz}$. The antenna is to be designed on substrate which has a relative permittivity $\varepsilon_{\mathrm{r}}$ of 2.54 , a dielectric thickness $\mathrm{h}$ of $1.6 \mathrm{~mm}$, a loss tangent of about 0,019 and 0.05 $\mathrm{mm}$ conductor thickness. A rectangular slot with $\mathrm{L}_{\mathrm{ap}}=26 \mathrm{~mm}$ and width $\mathrm{W}_{\mathrm{ap}}=2.6 \mathrm{~mm}$ is used for coupling the patch to a microstrip line of length $\mathrm{L}_{\mathrm{f}}=20 \mathrm{~mm}$, etched on substrate witch has a relative permittivity $\varepsilon_{\mathrm{r}}$ of 2.54 , a dielectric thickness h of $1.6 \mathrm{~mm}$, a loss tangent of about 0.019 and $0.05 \mathrm{~mm}$ conductor thickness. A single coaxial probe is connected directly to the antenna through a microstrip line (SMA connector).

The mask of the bow-tie antenna with dimensions is shown in the figure below.

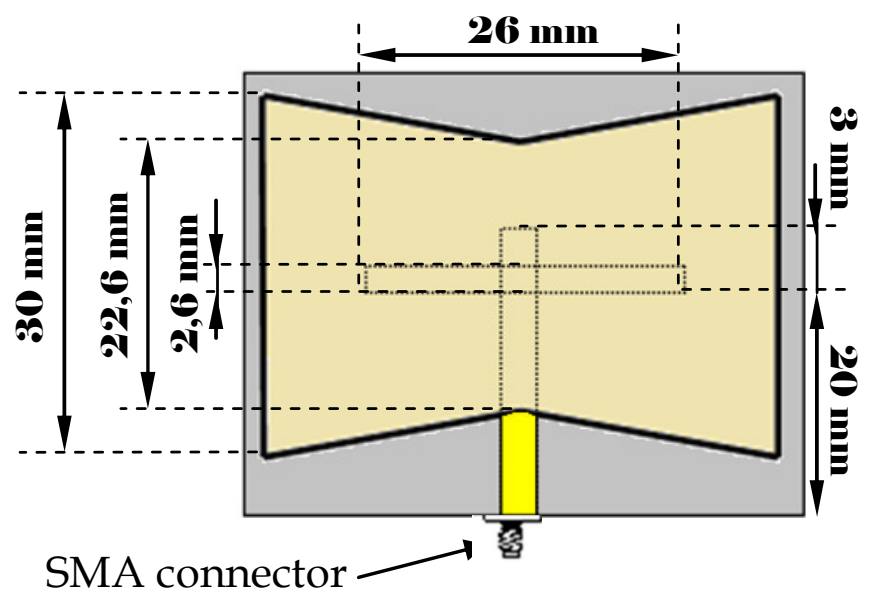

Figure 4. Mask of the multilayered bow-tie antenna operating at the frequency $2.4 \mathrm{GHz}$. 
International Journal of Computer Networks \& Communications (IJCNC) Vol.4, No.3, May 2012

The simulated input return loss of bow-tie antenna is displayed at the frequency $2.4 \mathrm{GHz}$ in figure 5 .

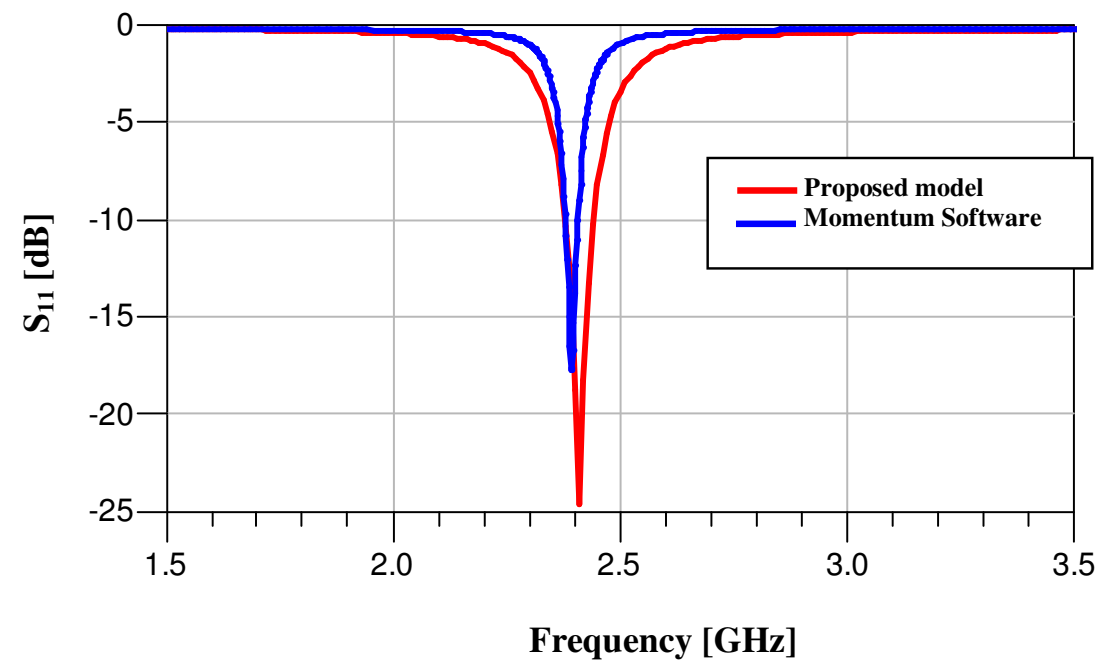

Figure 5. Simulated input antenna return loss.

At the frequency $2.4 \mathrm{GHz}$, we observe appearance of a resonant mode and a good matching by the proposed model and the Momentum software. It appears a peak of $-24.62 \mathrm{~dB}$ using transmission line model with a light shift by the moment method. At the frequency $2.39 \mathrm{GHz}$ we recorded a peak of $-17.68 \mathrm{~dB}$.

The Moments results and those obtained from transmission line model of the input phase of return loss for this antenna is shown in figure 6.

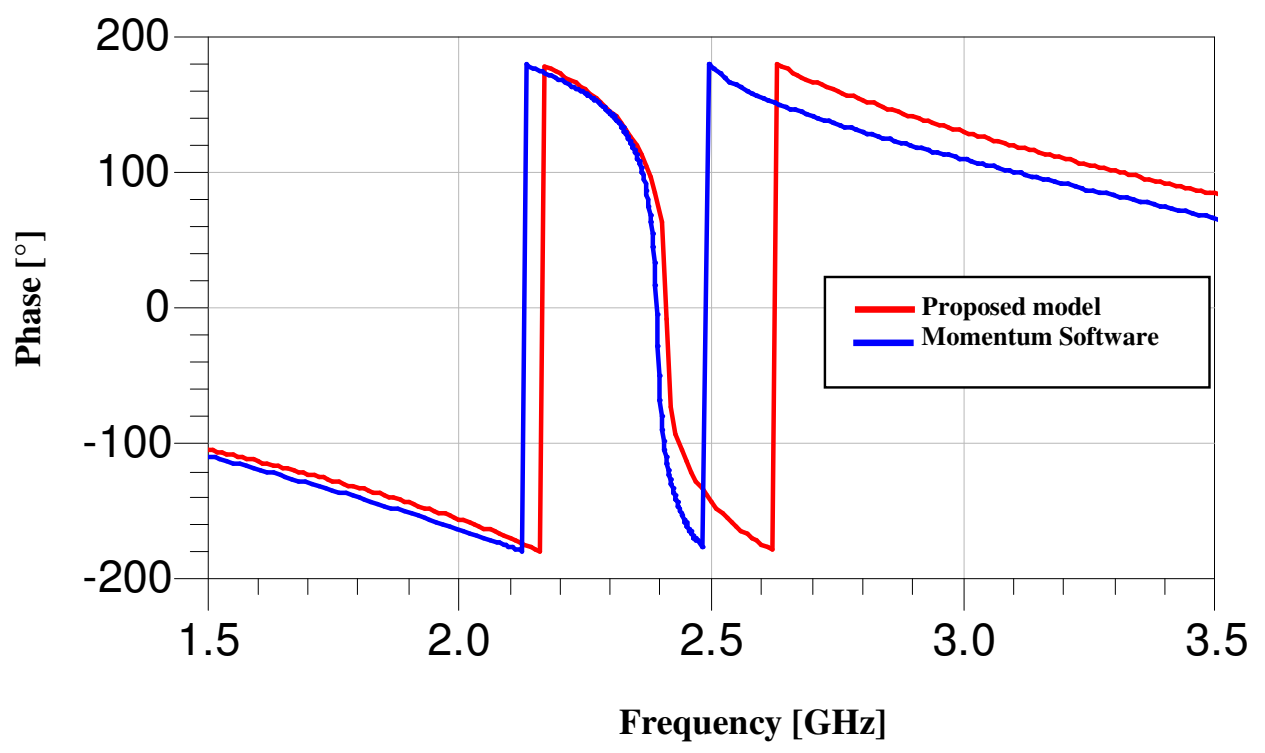

Figure 6. Reflected phase at the antenna input. 
International Journal of Computer Networks \& Communications (IJCNC) Vol.4, No.3, May 2012

We note very well that the phase is null by the two models at the resonant frequencies, which means a perfect adaptation. The simulated VSWR is represented on the figure below.

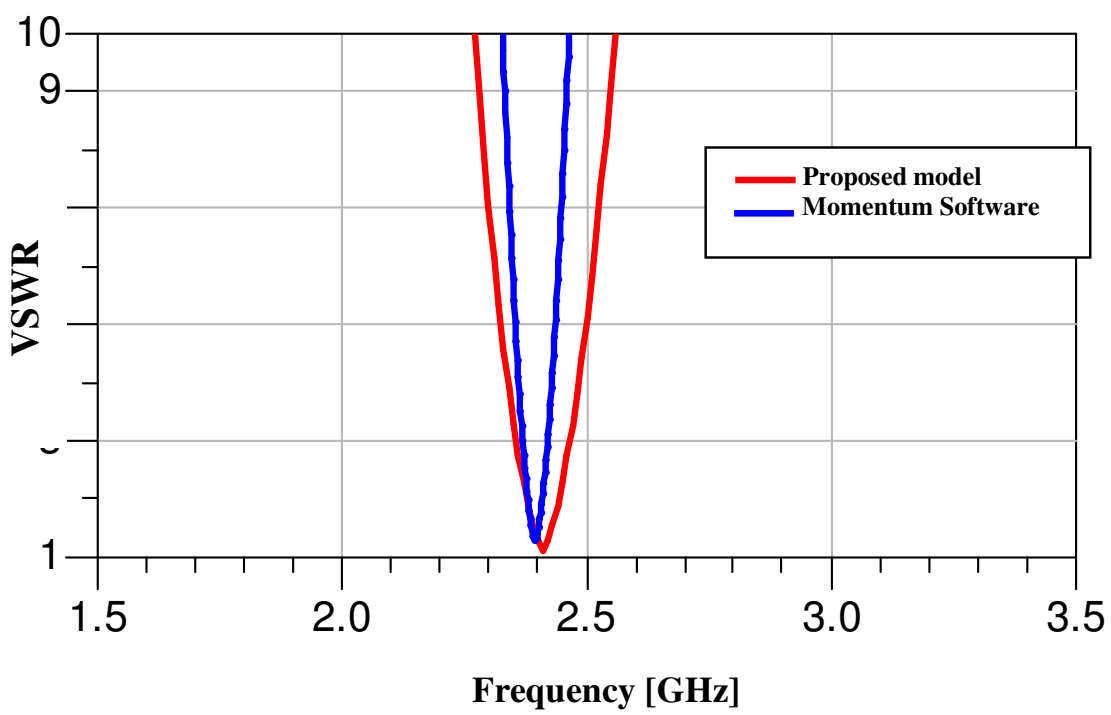

Figure 7. Simulated input antenna VSWR.

According to the figure 7, we note a perfect similarity between the two curves. In the vicinity of the resonant frequency, the VSWR is close to unity.

The impedance locus of the bow-tie antenna is illustrated on Smith's chart in figure 8 .

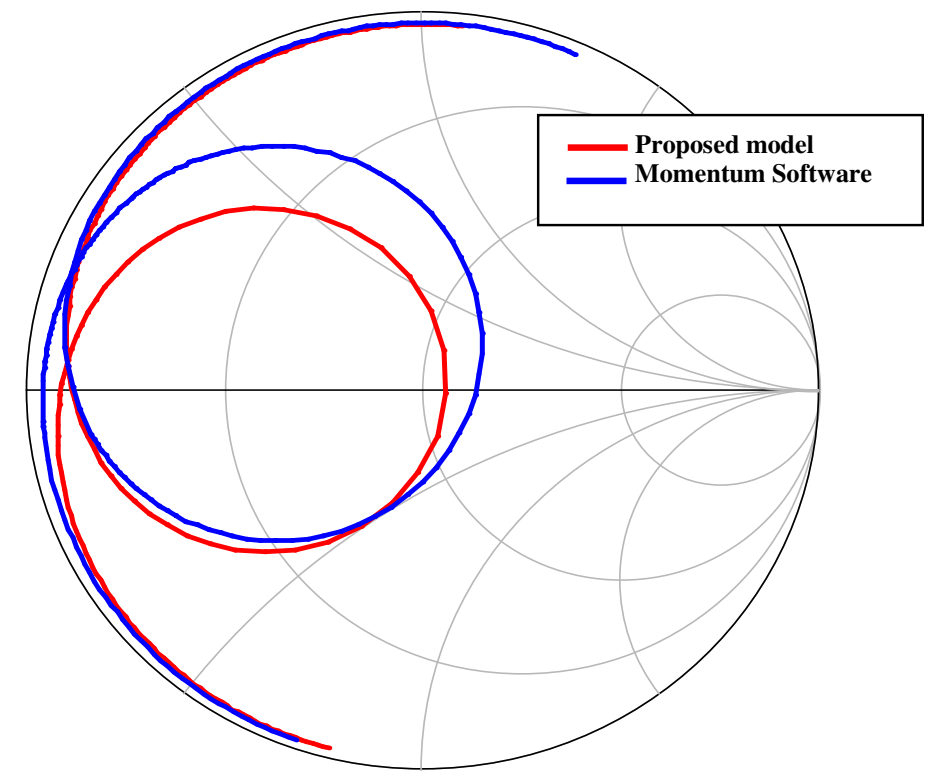

Figure 8. Smith's chart of the input impedance return losses. 
International Journal of Computer Networks \& Communications (IJCNC) Vol.4, No.3, May 2012

It can be seen from Figure 8 that the comparison for the input impedance between transmission line model and the moment method results are in good agreement. One notices that the resonant frequency is very close to the axis of $50 \mathrm{Ohm}$.

\section{2. Bow-tie antenna multi-layer operating at the resonant frequency $8 \mathrm{GHz}$}

The antenna is to be designed on substrate which has a relative permittivity $\varepsilon_{\mathrm{r}}$ of 2.54 , a dielectric thickness $\mathrm{h}$ of $1.6 \mathrm{~mm}$, a loss tangent of about 0,019 and $0.05 \mathrm{~mm}$ conductor thickness. A rectangular slot with $\mathrm{La}=16 \mathrm{~mm}$ and width $\mathrm{W}_{\mathrm{a}}=2.6 \mathrm{~mm}$ is used for coupling the microstrip line of $10 \mathrm{~mm}$ length to the patch, etched on a substrate which has a relative permittivity $\varepsilon_{\mathrm{r}}$ of 2.54 , a dielectric thickness $\mathrm{h}$ of $1.6 \mathrm{~mm}$, a loss tangent of about 0,019 and 0.05 $\mathrm{mm}$ conductor thickness. The antenna was designed to operate with a resonant frequency of 8 GHz. A single coaxial probe is connected directly to the antenna through a microstrip line (SMA connector).

The bow-tie antenna architecture with dimensions in millimetre is represented in figure 9.

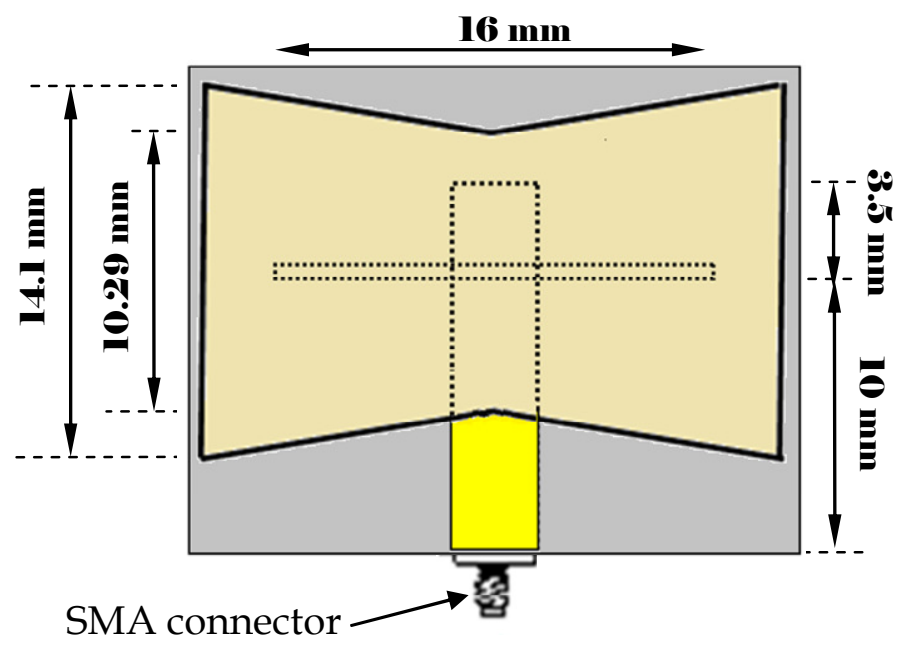

Figure 9. Mask of the bow-tie antenna multilayer operating at the frequency $8 \mathrm{GHz}$.

The simulated input return loss of bow-tie antenna is displayed at the frequency $8 \mathrm{GHz}$ in figure 10. 


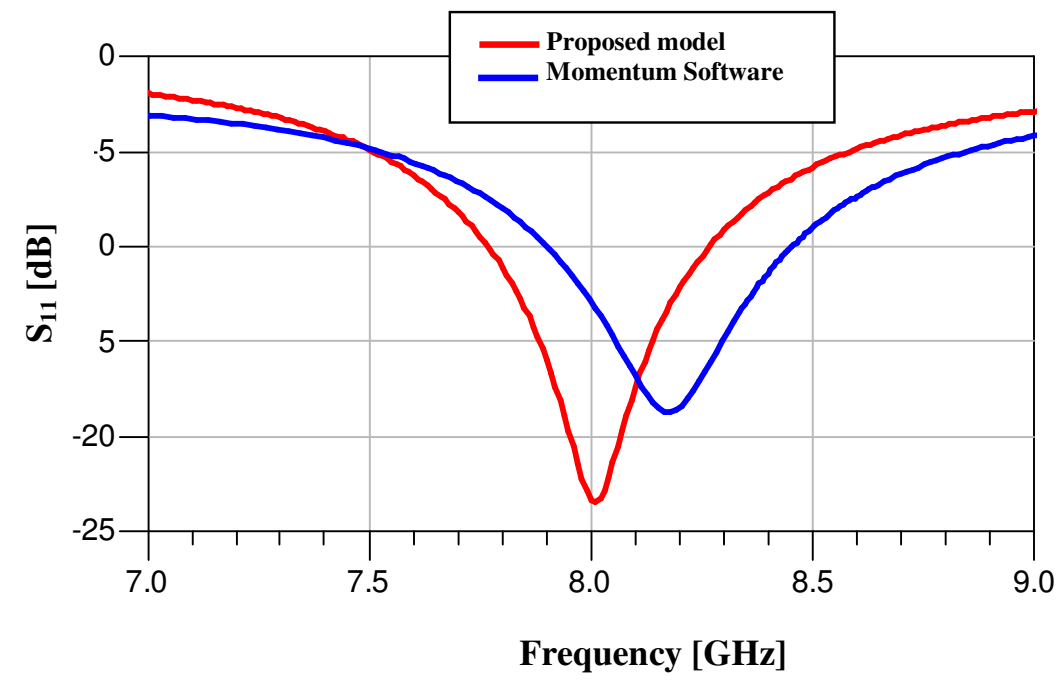

Figure 10. Simulated input antenna return loss.

From figure 10, we observe that the resonance of the antenna is correctly predicted to $8 \mathrm{GHz}$ with a peak of $-23.46 \mathrm{~dB}$ in transmission line model; there is light shift at resonant frequency by the moment's method with a peak of $-18.71 \mathrm{~dB}$.

Reflected phases obtained by the two models are illustrated in figure 11.

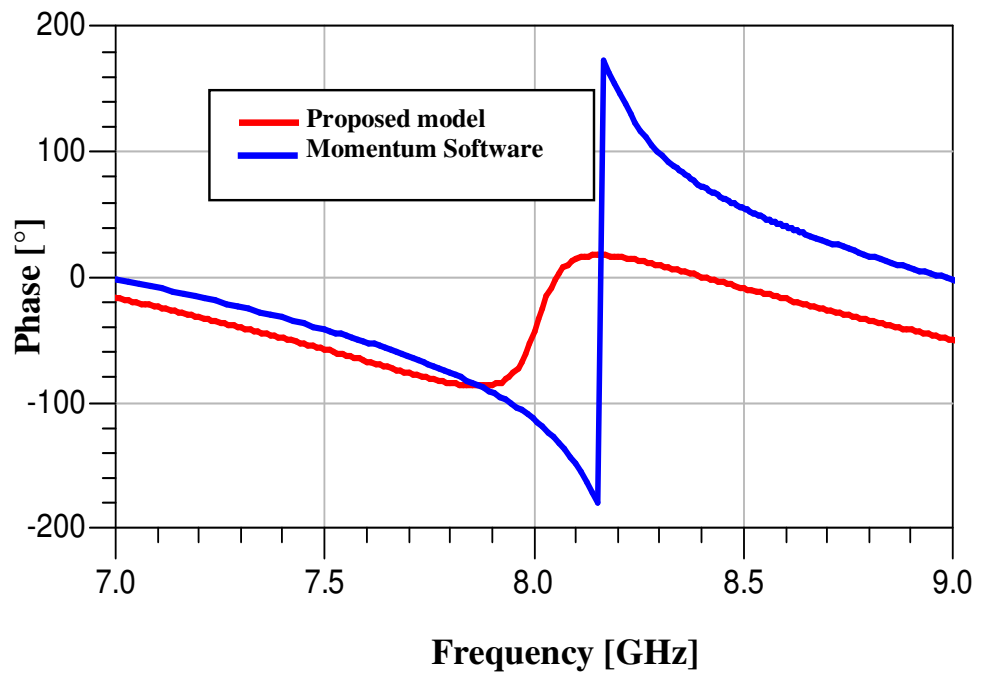

Figure 11. Reflected phase at the antenna input.

We note that there is a good agreement between the transmission line model and moment's method. The simulated VSWR at the frequency $8 \mathrm{GHz}$ is represented on the figure below. 
International Journal of Computer Networks \& Communications (IJCNC) Vol.4, No.3, May 2012

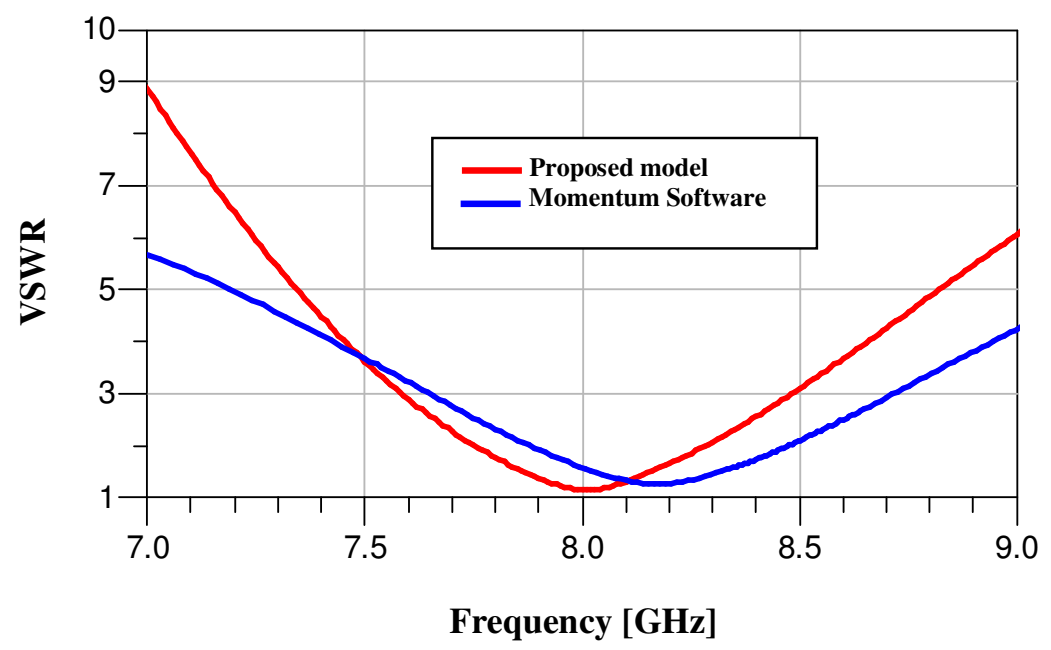

Figure 12. Simulated input antenna VSWR.

We observe that the VSWR level is close to unity for the both models (model of the transmission line and the method of moments), which implies a good adaptation of the antenna.

The impedance locus of the multi-layers bow-tie antenna at the frequency $8 \mathrm{GHz}$ is illustrated on Smith's chart in figure 13.

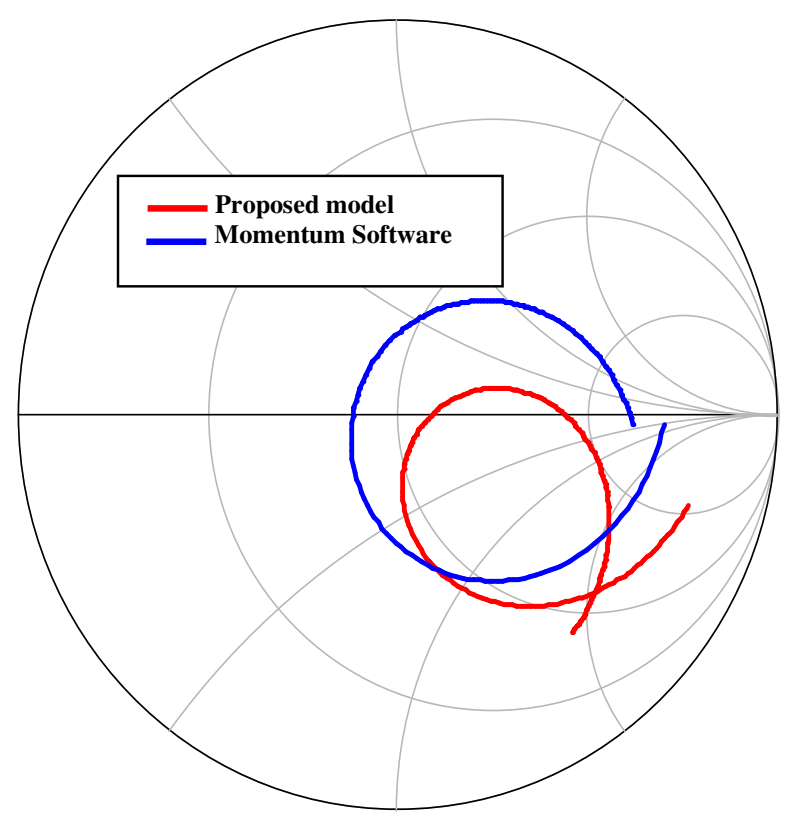

Figure 13. Smith's chart of the input impedance returns losses. 
International Journal of Computer Networks \& Communications (IJCNC) Vol.4, No.3, May 2012

It can be seen from figure 13 that the comparison for the input impedance between transmission line model and the moment method results are in good agreement. We note that the resonant frequency is very close to the axis of $50 \mathrm{Ohm}$.

\section{CONCLuSion}

In this paper, two multilayer bowtie antennas are designed to resonate at the frequencies 2.4 $\mathrm{GHz}$ and $8 \mathrm{GHz}$ corresponding to RFID and Radars applications using an equivalent circuit. The proposed transmission line model showed its interest in the design of different multilayered bow-tie antennas predict the correct resonance frequency for different applications in telecommunications.Even though the model is conceptually simple, it still produces accurate results in a relatively short period of computing time. The results obtained highlighted an excellent agreement between the transmission line model and the moment's method.

\section{REFERENCES}

[1] M. Abri, N. Boukli-hacene, F. T. Bendimerad, E. Cambiaggio "Design of a Dual Band Ring Printed Antennas Array” Microwave journal., Vol. 49, N. 5, pp. 228-232, 2006.

[2] M. Abri, F. T. Bendimerad., N. Boukli-hacene and M. Bousahla, 'A Log Periodic Series-Fed Antennas Array Design Using a Simple Transmission Line Model'., International Journal of Electronics and Communication Engineering ISSN 0974-2166 Volume 2, Number 3 (2009), pp. 161-169.

[3] M. Abri, H. Dib and A. S. E. Gharnaout, 'Accurate Model for Single Bow-Tie Antenna Design', International Journal of Microwave And Optical Technology, VOL.6, N.5, 2011.

[4] J. George, M. Deepukumar, C. K. Aanandan, P. Mohanan, and K. G. Nair, "New compact microstrip antenna,” Electronics Letters, vol. 32, pp. 508-509,1996.

[5] Uduwawala, D. et al., "A deep parametric study of resistor-loaded bowtie antennas for ground penetrating radar applications using FDTD," IEEE Trans. Geosciences and Remote Sensing, vol. 42, No. 4, pp. 732- 742, Jun. 2004.

[6] Birch, M. and Palmer K. D., "Optimized bowtie antenna for pulsed low frequency groundpenetrating radar,” Proceeding of SPIE, vol. 4758, 2002.

[7] George, J. and al.: "New compact microstrip antenna”, Electron. Lett., 1996, 32, pp. 508-509.

[8] K. R. Mahmoud., "Design optimization of a bow-tie antenna for $2.45 \mathrm{GHz}$ RFID readers using a hybrid BSO-NM algorithm,“ Progress In Electromagnetics Research, PIER 100, 105-117, 2010.

[9] Sener Uysal, Mook-Seng Leong, and Chee Hong Ng, "Bowtie Patch Antennas and Simple Arrays for Wireless Indoor Communications", IEEE transactions on microwave theory and techniques, Vol. 47, No. 6, June 1999.

[10] C. A. Balanis, Antenna Theory Analysis and Design, Second Edition, John Wiley and Sons, 1997.

[11] Himdi, M., Daniel, J. P., and C. Terret, "Transmission line analysis of aperture-coupled microstrip antenna", Electronics Letters, vol. 25, no. 18, Aug. 1989, pp. 1229-1230. 